\title{
Friedreich's Ataxia and Auditory Processing Disorder
}

\author{
Helio AG Teive ${ }^{1}$ - Vasiliki Maria Iliadou ${ }^{2}$ Mario Manto ${ }^{3,4}$ \\ Published online: 18 February 2021 \\ (C) The Author(s), under exclusive licence to Springer Science+Business Media, LLC part of Springer Nature 2021
}

Friedreich's ataxia (ATX-FXN) was described by Nikolaus Friedreich's in 1863 and is the most common autosomal recessive ataxia worldwide [1-3]. The estimated carrier frequency in the general population is $1 / 60$ to $1 / 90$ and prevalence of the disease has been estimated in a variety of Western European populations between 1:20,000 and 1:125,000 [1-3]. In the large majority of cases (95\%), the disease is caused by a pathological homozygous repeat expansion of a trinucleotide repeat (GAA: usually between 100 and 1300 repeats) in intron 1 of the $\mathrm{X} 25$ frataxin $(\mathrm{FXN})$ gene on chromosome 9q13 [1-3]. The severity of the phenotype correlates with the size of the GAA expansion. The mutation causes a defect of transcription and lack of FXN, a small mitochondrial protein, resulting in a complex neurological presentation. Although the exact function of FXN is still a matter of discussion, there is general agreement that the protein is involved in cellular iron homeostasis and that its deficiency results in multiple enzyme deficits, mitochondrial dysfunction, and oxidative damage $[1,2]$.

The "classical" phenotype of ATX-FXN is characterized by progressive gait and limb ataxia, dysarthria, loss of lower limb reflexes with deep sensory loss, Babinski's sign, eye movement abnormalities, particularly square-wave jerks, hypermetric saccades and saccadic pursuit, optic atrophy, and the presence of extra-neurological signs including scoliosis, pes cavus, foot deformities, hypertrophic cardiomyopathy, and diabetes mellitus [1-3]. At least 35\% patients present with

Mario Manto

mario.manto@ulb.be

1 Movement Disorders Unit, Neurology Service, Internal Medicine Department, Hospital de Clínicas, Federal University of Paraná, Curitiba, Paraná, Brazil

2 Clinical Psychoacoustics Lab, 3rd Psychiatric Department, Neurosciences Sector, School of Medicine, Faculty of Health Sciences, Aristotle University of Thessaloniki, Thessaloniki, Greece

3 Unité des Ataxies Cérébelleuses, Service de Neurologie, CHU-Charleroi, Charleroi, Belgium

4 Service des Neurosciences, Université de Mons, Mons, Belgium atypical clinical picture (variants), including LOFA (late-onset cerebellar ataxia) and VLOFA (very late-onset cerebellar ataxia). Delayed-onset forms are characterized by a less severe phenotype, a slower progression, retained reflexes, or even hyperreflexia and spasticity. Rare cases have been described presenting with chorea, dystonia, and myoclonus [1-4]. In addition to a better identification of the various phenotypic presentations, neuroimaging studies have expanded our understanding of the repercussions of the disease on the central nervous system $[1,2]$. Magnetic resonance imaging (MRI) was used to diagnose ATX-FXN before gene testing was available, and thinning of the cervical spinal cord was a consistent observation $[2,5,6]$. Atrophy of the cerebellum and brainstem was more variable, but in structural exams performed in recent years, the images have clearly revealed atrophy of the dentate nuclei and degeneration of the superior cerebellar peduncles $[2,5,6]$. Diffusion tensor imaging (DTI) and tract-based spatial statistics (TBSSS) also clearly demonstrate the extensive cerebral and cerebellar involvement in ATX-FXN [5]. A multimodal MRI-based study has investigated the anatomical substrates involved in the early stages of ATX-FXN and suggests that structural damage begins in pediatric patients in the spinal cord and inferior cerebellar peduncle, as well the red nucleus, and progresses to widespread cerebral areas in adulthood [6]. These findings contribute to our understanding of the different clinical phenotypes of the disease.

Several studies have demonstrated impaired vestibular functions in ATX-FXN, detected by caloric tests, rotatory tests, and head impulse tests (HIT) [7, 8]. Histopathological studies have revealed gliosis in the vestibular nucleus [9] and abnormalities of the vestibular nerve [10]. Up to $10 \%$ of patients complain of hearing loss [8]. Defective central auditory processing is common in ATX-FXN and can promote difficulties in verbal communication $[11,12]$. Conventional tests such as brainstem auditory evoked potentials (BAEPs) show increased latencies or dys-synchronization in waves I, III, and V, and in delays the inter-peak intervals I-III, I-V, and III-V [12]. Wave $\mathrm{V}$ may be undetectable. These findings confirm 
alterations in the integrity of the brainstem auditory pathways. Pure tone audiometry and acoustic immittance measures may be impaired also. Damage to the cochlear nerve without involvement to inner ear structures has been reported [10], with spiral ganglion degeneration [13]. The auditory neuropathy of ATX-FXN might be related to a mechanism of fast energetic failure [14]. Auditory perception is commonly abnormal in ATX-FXN if investigated correctly. What we tend to think as normal hearing (the pure tone audiogram) is limited to normal hearing sensitivity. Holistically evaluating hearing abilities in any individual requires testing for hearing sensitivity as well as for auditory processing abilities [15]. While there are studies showing deficits in auditory processing in ATXFXN, the systematic implementation of an auditory processing battery that provides a good view to hearing is rarely the case. This issue of Cerebellum includes an innovative paper entitled "Auditory phenotypic variability in Friedreich's ataxia patients" by Prof Bamiou's team linking auditory processing disorder (APD) with genotype as well as cognition [16]. Implications include (i) early identification and appropriate management of APD may have a positive effect on cognition by ameliorating deficits that have a negative effect on the quality of life, and (ii) auditory deprivation should not be solely thought as an abnormal audiogram as hearing encompasses many facets. The link between auditory deprivation (in this case of the form of APD) and cognition has long-term outcomes and should not be thought as a cross-sectional one $[17,18]$. Thus, it is of outmost importance to longitudinally evaluate patients with ATX-FXN, document the cognitive deficits when identifying APD at an early stage, and provide appropriate management for these challenging patients. Could this improve patients' quality of life? This remains to be confirmed. Furthermore, the issue of a possible use of these findings as a biomarker for longitudinal or therapeutic studies remains open and requires novel studies.

\section{References}

1. Bürk K. Friedreich's ataxia: current status and future prospects. Cerebellum Ataxias. 2017;4(4).

2. Cook A, Giunti P. Friedreich's ataxia: clinical features, pathogenesis and management. Br Med Bull. 2017;124:19-30.
3. Beaudin M, Matilla-Duenas A, Soong BW, et al. The classification of autosomal recessive cerebellar ataxias: a consensus statement from the Society for Research on the Cerebellum and Ataxias Task Force. Cerebellum. 2019;18:1098-125.

4. Hou JGG, Jankovic J. Movement disorders in Friedreich's ataxia. J Neurol Sci. 2003;206(1):59-64.

5. Karuta SCV, Raskin S, Carvalho Neto A, Gasparetto EL, Doring T, Teive HAG. Diffusion tensor imaging and tract-based spatial stastistics analysis in Friedreich's ataxia patients. Parkinsonism Relat Disord. 2015;21(5):504-8.

6. Rezende TJR, Martinez ARM, Faber I, Girotto Takazaki KA, Martins MP, Lima FD, et al. Developmental and neurodegenerative damage in Friedreich's ataxia. Eur J Neurol. 2019;26(3):483-9.

7. Prasher DK, Cohen M. The selective effects of central masking on brain stem potentials. Br J Audiol. 1984;18(2):79-83.

8. Fahey MC, Cremer PD, Aw ST, Millist L, Todd MJ, White OB, et al. Vestibular, saccadic and fixation abnormalities in genetically confirmed Friedreich ataxia. Brain. 2008;131(Pt 4):1035-45.

9. Oppenheimer DR. Brain lesions in Friedreich's ataxia. Can J Neurol Sci. 1979;6(2):173-6.

10. Spoendlin H. Optic cochleovestibular degenerations in hereditary ataxias. II. Temporal bone pathology in two cases of Friedreich's ataxia with vestibulo-cochlear disorders. Brain. 1974;97(1):41-8.

11. Rance G, Corben LA Du Bourg E, et al. Successful treatment of auditory perceptual disorder in individuals with Friedreich's ataxia. Neuroscience. 2010;171:552-5.

12. Zeigelboim BS, Teive HAG, da Rosa MR, et al. The importance of central auditory evaluation in Friedreich's ataxia. Arq Neuropsiquiatr. 2018;76(3):170-6.

13. Satya-Murti S, Cacace A, Hanson P. Auditory dysfunction in Friedreich ataxia: result of spiral ganglion degeneration. Neurology. 1980;30(10):1047-53.

14. Giraudet F, Charles P, Mom T, Boespflug-Tanguy O, Dürr A, Deltenre $\mathrm{P}$, et al. Rapid exhaustion of auditory neural conduction in a prototypical mitochondrial disease, Friedreich ataxia. Clin Neurophysiol. 2018;129(6):1121-9.

15. Iliadou VV, Ptok M, Grech H, Pedersen ER, Brechmann A, Deggouj N, et al. A European perspective on auditory processing disorder-current knowledge and future research focus. Front Neurol. 2017;8:622.

16. Koohi N, Thomas-Black G, Giunti P, Bamiou DE. Auditory phenotypic variability in Friedreich's ataxia patients. Cerebellum, in Press

17. Iliadou V, Moschopoulos N, Sidiras C, Eleftheriadou A, Nimatoudis I. Over-diagnosis of cognitive deficits in psychiatric patients may be the result of not controlling for hearing sensitivity and auditory processing. Psychiatry Clin Neurosci. 2018;72(9): 742 .

18. Johnson JCS, Marshall CR, Weil RS, Bamiou DE, Hardy CJD, Warren JD. Hearing and dementia: from ears to brain. Brain, 2020:awaa429. https://doi.org/10.1093/brain/awaa429.

Publisher's Note Springer Nature remains neutral with regard to jurisdictional claims in published maps and institutional affiliations. 